\title{
Epidemiological and Clinical Parameters Features of Patients with Clonorchiasis in the Geum River Basin, Republic of Korea
}

\author{
Hee-Eun Shin, ${ }^{1}$ Myoung-Ro Lee, ${ }^{1}$ Jung-Won Ju, ${ }^{1}$ Byong-Suk Jeong, ${ }^{1}$ Mi-Yeoun Park, \\ Keoung-Sook Lee, ${ }^{2}$ and Shin-Hyeong Cho ${ }^{1}$ \\ ${ }^{1}$ Division of Malaria and Parasitic Diseases, Center for Immunology and Pathology, National Research Institute of Health, \\ Centers for Disease Control and Prevention, Osong 28159, Republic of Korea \\ ${ }^{2}$ Department of Public Health, Okcheon-Gun Health Center, Okcheon 29032, Republic of Korea
}

Correspondence should be addressed to Shin-Hyeong Cho; he3un21@gmail.com

Received 23 November 2016; Accepted 16 February 2017; Published 26 April 2017

Academic Editor: Albert Eid

Copyright (C) 2017 Hee-Eun Shin et al. This is an open access article distributed under the Creative Commons Attribution License, which permits unrestricted use, distribution, and reproduction in any medium, provided the original work is properly cited.

This study was conducted to evaluate the infection rates of Clonorchis sinensis and laboratory findings in infected people. The 3,167 fecal samples, from nine villages in Okcheon-gun, were examined.

\section{Introduction}

Clonorchis sinensis is endemic in East Asian countries including China, Japan, Taiwan, Vietnam, and Korea $[1,2]$. Worldwide, it is currently estimated that 15-20 million people are infected and 1.5-2.0 million people show symptoms or complications [3-5]. Although a rapid decrease in the overall prevalence of intestinal parasites has been observed in Korea, the prevalence of $C$. sinensis has remained high in riverside areas [6]. Nationwide surveys reported C. sinensis infection rates of $4.6 \%$ in $1971,1.8 \%$ in $1976,2.6 \%$ in $1981,2.7 \%$ in 1986 , $2.2 \%$ in $1992,1.4 \%$ in $1997,2.4 \%$ in 2004 , and $1.9 \%$ in 2012 [7, 8]. Infection with the snail-transmitted trematode has been particularly frequent along several major Korean rivers, including the Geum, Nakdong, and Seomjin [9]. The rates of C. sinensis infection observed in the Geum River basin were $12.0 \%$ in 1981 [9], $4.6 \%$ in 2006 [10], and $3.1 \%$ in 2007 [11].

Freshwater fish is well-known as the major second intermediate host for $C$. sinensis, which can infect humans who ingest infected fish that is raw or undercooked [10]. The prevalence of infection is higher in males and increases with age in Korea [12]. Clonorchiasis patients suffer from symptoms such as epigastric pain, abdominal tenderness, fever, jaundice, and diarrhea [3]. Moreover, chronic infection with C. sinensis may cause more severe diseases such as cholangiocarcinoma [13]. The presence of the worms in the walls of the smaller bile ducts causes necrosis, inflammation, and biliary stricture through several mechanisms [14].

Early diagnosis and treatment are very important in preventing complications from clonorchiasis. The diagnosis depends mostly on detection of eggs in a patient's feces by either the formalin-ether concentration technique (FECT) or the Kato-Katz thick smear method [15]. Although molecular diagnostic techniques have been developed, early diagnosis of clonorchiasis remains difficult because subclinical infection with C. sinensis often occurs without obvious signs. Laboratory blood tests to look for changes in overall health are often part of a regular medical checkup. Individuals with abnormal blood test results should be further examined with a test suitable for early diagnosis. Previous studies reported that $C$. sinensis infected groups had differences in their serum alkaline phosphatase (ALP), aspartate aminotransferase (AST), alanine aminotransferase (ALT), $\gamma$-glutamyltranspeptidase ( $\gamma$-GTP), and alpha-fetoprotein (AFP) test results and presented with peripheral eosinophilia $[2,16]$. However, there is no diagnostic set of results from common blood and biochemical tests, except for those tests that can indicate compromised liver function.

In this study, we investigated sociodemographic and behavioral risk factors for $C$. sinensis infection in inhabitants living near the Geum River. We additionally evaluated the 
(a)

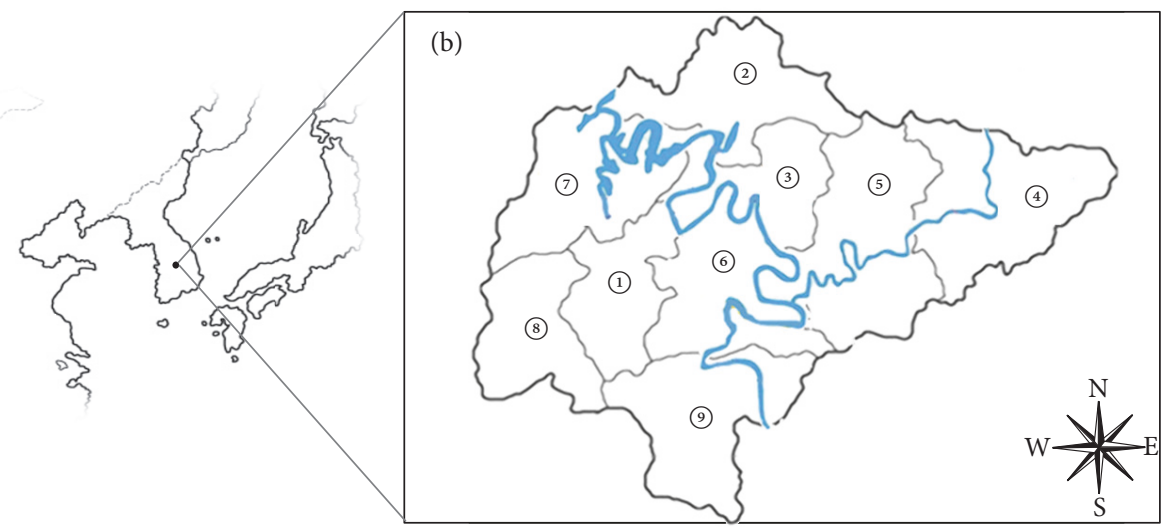

Figure 1: Geographic locations of the surveyed areas. Map shows (a) the Korean Peninsula and (b) the Okcheon-gun County of the Chungcheongbuk-do Province of Korea. The Geum River is shown in blue. Individual villages are shown on the map as follows: (1) Okcheoneup, (2) Annae-myeon, (3) Annam-myeon, (4) Cheongsan-myeon, (5) Cheongseong-myeon, (6) Dongi-myeon, (7) Gunbuk-myeon, (8) Gunseomyeon, and (9) Iwon-myeon.

results of subjects' laboratory blood tests in order to look for possible early diagnostic indicators of clonorchiasis.

\section{Materials and Methods}

2.1. Study Site. The study was undertaken during the period of January to December 2013. A total of 3,167 inhabitants (1,227 males and 1,940 females) from nine villages in the Okcheon-gun County of the Chungcheongbuk-do Province of Korea were examined (Figure 1). The ages of the inhabitants ranged from 9 to 99 years, and the average age was 68.4 years (Table 1).

2.2. Fecal Examination. Stool specimens were collected in plastic stool containers and transferred to the laboratory of the Korea National Research Institute of Health during the period from February through May 2013. All participants were recruited for stool collection by a random household sampling method. Stool specimens were examined using the formalin-ether concentration technique (FECT) to assess the prevalence of parasitic infection, and the eggs per gram (EPG) were calculated using Stoll's egg counting method. Patients who tested positive for eggs (egg-positive) were considered infected, and those who tested negative for eggs (eggnegative) were considered uninfected.

2.3. Laboratory Examination. Blood samples ( $5 \mathrm{~mL}$ per individual) were collected from 97 participants using both 2-[2-[bis(carboxymethyl)amino] ethyl-(carboxymethyl)amino]acetic acid (EDTA) coated tubes and serum-separating tubes (SSTs). Samples were examined using 15 hematological parameters: hemoglobin $(\mathrm{Hb})$, hematocrit $(\mathrm{Hct})$, red blood cell count (RBC), white blood cell count (WBC), platelet distribution width (PDW), platelet counts, mean corpuscular volume $(\mathrm{MCV})$, mean corpuscular hemoglobin $(\mathrm{MCH})$, mean corpuscular hemoglobin concentration (MCHC), percent eosinophils (EOS), percent basophils, percent monocytes, percent neutrophils (NEUT), percent lymphocytes, and mean plasma volume (MPV). In addition, we analyzed 18 biochemical parameters using an automatic blood analyzer (Olympus AU 400): AST, ALT, lactate dehydrogenase (LDH), ALP, creatine phosphokinase (CPK), $\gamma$-GTP, total protein, albumin, total cholesterol, high-density lipoprotein (HDL), low-density lipoprotein (LDL), triglycerides (TG), total bilirubin, direct bilirubin, blood urea nitrogen (BUN), creatinine, uric acid (UA), and random blood sugar (RBS).

2.4. Questionnaire Survey. We questioned all participants to identify correlations between C. sinensis infection and known risk factors. The questionnaire was given to all participants verbally during an interview by a trained worker in a public health center. The following general information was collected: participant gender, age, current residence, and whether they consumed alcohol. We also asked questions about clonorchiasis risk-related behaviors: Did the patient have a history of eating raw freshwater fish, and if so when, where was the fish from, and what species of fish was consumed? Did the patient have a previous history of C. sinensis infection, or of bile duct or liver diseases, or of cooking freshwater fish? Had the patient ever experienced symptoms such as anorexia, indigestion, jaundice, abdominal discomfort, or anemia?

2.5. Statistical Analysis. Statistical analyses were performed using the SPSS 18.0 program (ver. 18.0; Chicago, Illinois, USA). Only participants with complete records were included in the final analysis. A chi-square test and independent $t$ test were used to check for statistically significant differences between questionnaire answers from the infected versus noninfected groups. In addition, we confirmed that the differences between the groups' laboratory results for peripheral blood cells were statistically significant, by an independent $t$ test. Differences were considered statistically significant when $p$ values were less than $0.05(p<0.05)$. Finally, a Pearson correlational analysis was performed to compare EPG to each of the laboratory test results for infected patients. 
TABLE 1: Distribution of the participants.

\begin{tabular}{|c|c|c|c|c|c|c|c|c|c|c|}
\hline \multirow{2}{*}{ Location } & \multirow{2}{*}{ Gender } & \multirow{2}{*}{ Number of inhabitants examined } & \multicolumn{8}{|c|}{ Age } \\
\hline & & & $0-19$ & $20-29$ & $30-39$ & $40-49$ & $50-59$ & $60-69$ & $70-79$ & $80 \leq$ \\
\hline \multirow{3}{*}{$\begin{array}{l}\text { Okcheon- } \\
\text { eup }\end{array}$} & Male & 213 & 4 & 3 & 8 & 13 & 28 & 42 & 90 & 25 \\
\hline & Female & 389 & 2 & 1 & 5 & 8 & 36 & 88 & 183 & 67 \\
\hline & Total & 602 & 6 & 4 & 13 & 21 & 64 & 130 & 273 & 92 \\
\hline \multirow{3}{*}{$\begin{array}{l}\text { Annae- } \\
\text { myeon }\end{array}$} & Male & 136 & 0 & 0 & 1 & 9 & 23 & 42 & 48 & 13 \\
\hline & Female & 188 & 2 & 0 & 3 & 8 & 31 & 44 & 82 & 18 \\
\hline & Total & 324 & 2 & 0 & 4 & 17 & 54 & 86 & 130 & 31 \\
\hline \multirow{3}{*}{$\begin{array}{l}\text { Annam- } \\
\text { myeon }\end{array}$} & Male & 76 & 0 & 0 & 0 & 2 & 9 & 12 & 39 & 14 \\
\hline & Female & 145 & 0 & 0 & 1 & 1 & 14 & 27 & 78 & 24 \\
\hline & Total & 221 & 0 & 0 & 1 & 3 & 23 & 39 & 117 & 38 \\
\hline \multirow{3}{*}{$\begin{array}{l}\text { Cheong } \\
\text { san-myeon }\end{array}$} & Male & 162 & 0 & 0 & 1 & 6 & 25 & 50 & 70 & 10 \\
\hline & Female & 273 & 0 & 0 & 3 & 6 & 41 & 71 & 119 & 33 \\
\hline & Total & 435 & 0 & 0 & 4 & 12 & 66 & 121 & 189 & 43 \\
\hline \multirow{3}{*}{$\begin{array}{l}\text { Cheong } \\
\text { seong- } \\
\text { myeon }\end{array}$} & Male & 165 & 0 & 0 & 2 & 6 & 18 & 41 & 83 & 15 \\
\hline & Female & 236 & 0 & 0 & 0 & 4 & 28 & 58 & 103 & 43 \\
\hline & Total & 401 & 0 & 0 & 2 & 10 & 46 & 99 & 186 & 58 \\
\hline \multirow{3}{*}{$\begin{array}{l}\text { Dongi- } \\
\text { Myeon }\end{array}$} & Male & 146 & 0 & 1 & 6 & 11 & 22 & 29 & 61 & 16 \\
\hline & Female & 190 & 0 & 1 & 3 & 13 & 25 & 46 & 80 & 22 \\
\hline & Total & 336 & 0 & 2 & 9 & 24 & 47 & 75 & 141 & 38 \\
\hline \multirow{3}{*}{$\begin{array}{l}\text { Gunbuk- } \\
\text { Myeon }\end{array}$} & Male & 125 & 1 & 2 & 6 & 9 & 18 & 24 & 53 & 12 \\
\hline & Female & 175 & 2 & 3 & 2 & 11 & 15 & 40 & 79 & 23 \\
\hline & Total & 300 & 3 & 5 & 8 & 20 & 33 & 64 & 132 & 35 \\
\hline \multirow{3}{*}{$\begin{array}{l}\text { Gunseo- } \\
\text { myeon }\end{array}$} & Male & 79 & 0 & 0 & 1 & 3 & 11 & 22 & 33 & 9 \\
\hline & Female & 137 & 0 & 0 & 2 & 3 & 17 & 26 & 65 & 24 \\
\hline & Total & 216 & 0 & 0 & 3 & 6 & 28 & 48 & 98 & 33 \\
\hline \multirow{3}{*}{$\begin{array}{l}\text { Iwon- } \\
\text { myeon }\end{array}$} & Male & 125 & 2 & 1 & 4 & 4 & 24 & 28 & 48 & 14 \\
\hline & Female & 207 & 1 & 0 & 4 & 5 & 28 & 58 & 91 & 20 \\
\hline & Total & 332 & 3 & 1 & 8 & 9 & 52 & 86 & 139 & 34 \\
\hline \multirow{3}{*}{ Total } & Male & 1,227 & 7 & 7 & 29 & 63 & 178 & 290 & 525 & 128 \\
\hline & Female & 1,940 & 7 & 5 & 23 & 59 & 235 & 458 & 880 & 273 \\
\hline & Total & 3,167 & 14 & 12 & 52 & 122 & 413 & 748 & 1,405 & 401 \\
\hline
\end{tabular}

2.6. Ethical Aspects. The study protocol was approved by the Ethics Committee of the Korea National Research Institute of Health, Centers for Disease Control \& Prevention, Republic of Korea (2013-06EXP-04-R). All participants and local health center workers were informed of the study's purpose and procedures. Written informed consent for the research was obtained from all participants prior to enrolment. The participants found to have intestinal parasitic infections were treated with praziquantel (2-(cyclohexanecarbonyl)-3,6, 7,11b-tetrahydro-1H-pyrazino[2,1-a] isoquinolin-4-one) and other appropriate antiparasitic drugs at the end of the survey.

\section{Results}

3.1. Infection Status. During the study period, 3,167 fecal samples were examined by the formalin-ether concentration technique (FECT). The total intestinal parasite infection rate of was $12.2 \%$ (385 cases) including 379 single infections (353 with C. sinensis, 24 with Metagonimus yokogawai, four with Trichuris trichiura, two with Gymnophalloides seoi, and two with Giardia lamblia) and six coinfections (two or more parasite species in a single sample). Coinfections with $C$. sinensis and M. yokogawai represented $0.2 \%$ (six cases) of the total infections. The C. sinensis infection rate was $11.1 \%$, and the mean EPG value was $171.7 \pm 513.4$ (Table 2 ). The riveradjacent village of Dongyi-myeon had the highest infection rate $(20.8 \%$ with a mean EPG $262.4 \pm 774.5)$. The second highest infection rate was $14.0 \%$ (mean EPG $58.5 \pm 82.6$ ) in the village of Annam-myeon. The lowest infection rate was $6.0 \%$ (mean EPG $95.3 \pm 186.7$ ) and was observed in the Gunseomyeon village. The prevalence of $C$. sinensis infection was shown to be highest among patients aged $70-79$ years $(10.7 \%)$.

3.2. Basic Characteristic. The results of the fecal examinations are summarized in Table 3. The infection (egg-positive) rates were significantly higher in males $(20.3 \%, p<0.001)$ and 
TABLE 2: Infection rates of C. sinensis according to surveyed area.

\begin{tabular}{lccc}
\hline Villages & Number of inhabitants examined & Infected (\%) & Mean EPG \pm SD \\
\hline Okcheon-eup & 602 & $48(8.0)$ & $94.6 \pm 186.7$ \\
Annae-myeon & 324 & $42(13.0)$ & $123.8 \pm 267.6$ \\
Annam-myeon & 221 & $31(14.0)$ & $58.5 \pm 82.6$ \\
Cheongsan-myeon & 435 & $43(9.9)$ & $221.3 \pm 621.4$ \\
Cheongseong-myeon & 401 & $43(10.7)$ & $344.0 \pm 736.1$ \\
Dongi-myeon & 336 & $70(20.8)$ & $262.4 \pm 774.5$ \\
Gunbuk-myeon & 300 & $33(11.0)$ & $31.4 \pm 41.0$ \\
Gunseo-myeon & 216 & $13(6.0)$ & $95.3 \pm 186.7$ \\
Iwon-myeon & 332 & $30(9.0)$ & $136.2 \pm 200.6$ \\
Total & 3,167 & $353(11.1)$ & $171.7 \pm 513.4$ \\
\hline
\end{tabular}

TABLE 3: C. sinensis infection rates based on sociodemographic and behavioral characteristics.

\begin{tabular}{|c|c|c|c|c|}
\hline Variables & Infected (\%) & Uninfected (\%) & Total (\%) & $p$ value \\
\hline Number & $353(11.1)$ & $2,814(88.9)$ & $3,167(100)$ & \\
\hline Gender & & & & 0.000 \\
\hline Male & $249(20.3)$ & $978(79.7)$ & $1,227(100)$ & \\
\hline Female & $104(5.4)$ & $1,836(94.6)$ & $1,940(100)$ & \\
\hline Age (yr) & & & & 0.146 \\
\hline$\leq 49$ & $15(7.5)$ & $185(92.5)$ & $200(100)$ & \\
\hline $50-59$ & $55(13.3)$ & $358(86.7)$ & $413(100)$ & \\
\hline $60-69$ & 89 (11.9) & $659(88.1)$ & $748(100)$ & \\
\hline$\geq 70$ & $194(10.7)$ & $1,612(89.3)$ & $1,806(100)$ & \\
\hline Alcohol drinking & & & & 0.000 \\
\hline Yes & $211(15.5)$ & $1,152(84.5)$ & $1,363(100)$ & \\
\hline No & $142(7.9)$ & $1,662(92.1)$ & $1,804(100)$ & \\
\hline Raw freshwater fish consumption & & & & 0.000 \\
\hline Yes & $147(14.7)$ & $851(85.3)$ & $998(100)$ & \\
\hline No & $206(9.5)$ & $1,963(90.5)$ & $2,169(100)$ & \\
\hline History of cooking freshwater fish & & & & 0.000 \\
\hline Yes & $149(15.1)$ & $837(84.9)$ & $986(100)$ & \\
\hline No & $204(9.4)$ & $1,977(90.6)$ & $2,181(100)$ & \\
\hline Symptom experience & & & & 0.975 \\
\hline Yes & $126(11.2)$ & $1,002(88.8)$ & $1,128(100)$ & \\
\hline No & $227(11.1)$ & $1,812(88.9)$ & $2,039(100)$ & \\
\hline History of C. sinensis infection & & & & 0.061 \\
\hline Yes & $38(14.8)$ & $218(85.2)$ & $256(100)$ & \\
\hline No & $315(10.8)$ & $2,596(89.2)$ & $2,911(100)$ & \\
\hline History of bile ducts or liver disease & & & & 0.002 \\
\hline Yes & $31(18.3)$ & $138(81.7)$ & $169(100)$ & \\
\hline No & $322(10.7)$ & $2,676(89.3)$ & $2,998(100)$ & \\
\hline
\end{tabular}

alcohol drinkers $(15.5 \%, p<0.001)$ than in females $(5.4 \%)$ and nonalcohol drinkers (7.9\%), respectively. Based on the clonorchiasis risk-related behavioral questions, infection rates were significantly higher $(p<0.001)$ among the inhabitants who had eaten raw freshwater fish $(14.7 \%)$ or had cooked freshwater fish (15.1\%) than those who had not. In addition, significantly higher infection rates $(18.3 \%, p<0.01)$ were found among inhabitants whose anamneses included bile duct or liver related diseases than among those who reported no history of such diseases $(10.7 \%)$. However, the infection rates did not differ significantly based on patient age, history of $C$. sinensis infection, or symptom experience.

3.3. Correlation between C. sinensis Infection and Risk Factors. To assess the relationship between $C$. sinensis infection and risk factors, we selected factors that showed significant differences between infected and uninfected groups and then analyzed the results by logistic regression. The increased odds of infection (odds ratios (ORs)) for patients with various characteristics are shown in Table 4, along with their respective 
TABLE 4: Odds ratios (OR) and the 95\% confidence intervals (CIs) for C. sinensis infection for patients with different characteristics.



95\% confidence intervals (CIs). Males, alcohol drinkers, raw freshwater fish consumers, those who had cooked freshwater fish, and those with an anamnesis including bile duct or liver disease were $4.45,2.14,1.64,1.73$, and 1.87 times more likely to be infected, respectively.

3.4. Laboratory Findings. Among the hematological results, the MCV, MCHC, and the percentages of eosinophils (EOS) and neutrophils (NEUT) were significantly different $(p<$ 0.05 ) between the infected and uninfected groups. However, the RBC, WBC, platelet, basophil, monocyte, and lymphocyte counts were not significantly different nor were the PDW, $\mathrm{MCH}, \mathrm{MPV}, \mathrm{Hb}$, or Hct results. The biochemical tests revealed that $\mathrm{LDH}$ and ALP were mildly elevated, whereas the total protein, albumin, TG, and RBS were significantly decreased in the infected group. However, levels of AST, ALT, CPK, $\gamma$-GTP, total cholesterol, HDL, LDL, total bilirubin, direct bilirubin, BUN, creatinine, and uric acid were not significantly different between the groups (Table 5). The Pearson correlation analysis only revealed a statistically significant correlation ( $p=0.014$ ) between EPG and eosinophil count (Figure 2).

\section{Discussion}

We surveyed the characteristics and laboratory test results of patients with $C$. sinensis, living near the downstream portion of the Geum River basin. The overall prevalence of $C$. sinensis was $11.1 \%$ (353 cases). The infection rate was higher than previously reported. The prevalence of clonorchiasis in Geum River basin, for example, was reported to be $9.3 \%$ in 2002 [17] and 7.6\% in 2007 [11], respectively. Among the examined villages, the infection rate was high in inhabitants within $5 \mathrm{~km}$ of the riverside in areas such as Dongyi-myeon (20.8\%).

The clinical manifestations of the infection depend on the number of flukes, and the egg counts (EPG) give an indirect measure of the intensity of the parasitic infection. The EPG results in our study were lower than those previously reported from inhabitants of the Geum River basin in 1981 [9] and 2002 [17]. Our results indicate that over the last 30 years the infection intensity (EPG) has decreased, but infection rate has 




FIGURE 2: Relationship between parasite quantity (EPG) and eosinophil count in C. sinensis infected patients. Pearson correlation analysis shows a significant positive correlation in $C$. sinensis infected patients between the eggs per gram (EPG) in their stool and their eosinophil counts (Pearson's $r=0.36, p=0.014$, and $n=57$ ). The results of linear regression analysis are shown (line slope is $y=0.002 x+2.855$ and $r^{2}=0.13$ ).

increased. These results suggest that $C$. sinensis infections are still prevalent along the Geum River basin.

The general pattern of $C$. sinensis infection frequency shows a peak in the 50-59-year-old age group [10]. However, our results showed that the infection rate was highest in those aged 70-79 years. This peak in infection frequency among people in their seventies is likely to result from an accumulation of reinfection events or chronic infection with C. sinensis. The likelihood of C. sinensis infection was much higher for males than for females. In addition, the odds of infection were higher for alcohol drinkers, natural raw freshwater fish consumers, those who cooked freshwater fish, and those with a history of bile duct or liver diseases. Some of the results of our study are similar to those of previous researchers. In previous studies, the prevalence of $C$. sinensis has been higher in males, those who live in rural areas, those who have lived for long periods of time in riverside areas, and those who have eaten raw freshwater fish [4, 17-20].

The laboratory blood tests performed here are often included as part of a regular medical checkup to look for changes in patient health. In a previous report, laboratory tests showed eosinophilia with elevated ALP in a C. sinensis infected group, but other parameters such as AST, ALT, $\gamma$ GTP, and total bilirubin were not significantly affected [16]. Liao et al. (2006) demonstrated that ALP is increased in patients with liver or bile duct disease [21]. These results were similar to our findings that the mean ALP, LDH, and eosinophil counts were significantly increased. We also confirmed a correlation between infection intensity (EPG) and eosinophil counts. Among our 33 laboratory tests, the eosinophil count was the only test whose results significantly correlated with the EPG.

\section{Conclusions}

In conclusion, our report confirms that there are risk factors of C. sinensis infection and that several standard laboratory test results significantly differ between infected and uninfected groups. The inhabitants residing in Geum River basin should pay attention to clonorchiasis, and further work should be focused on the early diagnosis and treatment of patients with clonorchiasis because most patients infected with $C$. sinensis do not have symptoms.

\begin{tabular}{|c|c|}
\hline FECT: & Formalin-ether concentration technique \\
\hline EPG: & Eggs per gram \\
\hline EDTA: & $\begin{array}{l}\text { 2-[2-[bis(carboxymethyl)amino] ethyl- } \\
\text { (carboxymethyl)amino] acetic acid }\end{array}$ \\
\hline SST: & Serum-separating tube \\
\hline $\mathrm{Hb}:$ & Hemoglobin \\
\hline Hct: & Hematocrit \\
\hline RBC: & Red blood cell count \\
\hline WBC: & White blood cell count \\
\hline PDW: & Platelet distribution width \\
\hline MCV: & Mean corpuscular volume \\
\hline MCH: & Mean corpuscular hemoglobin \\
\hline MCHC: & $\begin{array}{l}\text { Mean corpuscular hemoglobin } \\
\text { concentration }\end{array}$ \\
\hline EOS: & Percent eosinophils \\
\hline BASO: & Percent basophils \\
\hline MONO: & Percent monocytes \\
\hline NEUT: & Percent neutrophils \\
\hline MPV: & Mean plasma volume \\
\hline Olympus AU 400: & Automatic blood analyzer \\
\hline AST: & Aspartate aminotransferase \\
\hline ALT: & Alanine aminotransferase \\
\hline LDH: & Lactate dehydrogenase \\
\hline ALP: & Serum alkaline phosphatase \\
\hline CPK: & Creatine phosphokinase \\
\hline$\gamma$-GTP: & $\gamma$-glutamyltranspeptidase \\
\hline HDL: & High-density lipoprotein \\
\hline LDL: & Low-density lipoprotein \\
\hline TG: & Triglycerides \\
\hline BUN: & Blood urea nitrogen \\
\hline UA: & Uric acid \\
\hline RBS: & Random blood sugar. \\
\hline
\end{tabular}

\section{Ethical Approval}

The study protocol was approved by the Ethics Committee of the Korea National Research Institute of Health, Centers for Disease Control \& Prevention, Republic of Korea (201306EXP-04-R). All participants and local health center workers were informed of the study's purpose and procedures.

\section{Consent}

Written informed consent for the research was obtained from all participants prior to enrolment.

\section{Conflicts of Interest}

The authors declare that they have no conflicts of interest. 


\section{Authors' Contributions}

Hee-Eun Shin, Byong-Suk Jeong, Mi-Yeoun Park, and ShinHyeong Cho conceived and designed the study. Hee-Eun Shin, Myoung-Ro Lee, Mi-Yeoun Park, Keoung-Sook Lee, and Shin-Hyeong Cho collected data. Hee-Eun Shin, JungWon Ju, and Shin-Hyeong Cho analyzed the data. Hee-Eun Shin and Shin-Hyeong Cho wrote the paper. All authors read and approved the final manuscript.

\section{Acknowledgments}

This work was supported by an Endemic Diseases Control Program (NIH 4800-4847-311) from the National Research Institute of Health, Centers for Disease Control and Prevention, Ministry of Health and Welfare, Republic of Korea.

\section{References}

[1] H.-J. Rim, “Clonorchiasis: an update," Journal of Helminthology, vol. 79, no. 3, pp. 269-281, 2005.

[2] K.-X. Wang, R.-B. Zhang, Y.-B. Cui, Y. Tian, R. Cai, and C.-P. Li, "Clinical and epidemiological features of patients with clonorchiasis," World Journal of Gastroenterology, vol. 10, no. 3, pp. 446-448, 2004.

[3] S. Hong and Y. Fang, "Clonorchis sinensis and clonorchiasis, an update," Parasitology International, vol. 61, no. 1, pp. 17-24, 2012.

[4] T. D. Thi Cam, A. Yajima, K. N. Viet, and A. Montresor, "Prevalence, intensity and risk factors for clonorchiasis and possible use of questionnaires to detect individuals at risk in northern Vietnam," Transactions of the Royal Society of Tropical Medicine and Hygiene, vol. 102, no. 12, pp. 1263-1268, 2008.

[5] M. B. Qian, Y. D. Chen, Y. Y. Fang et al., "Epidemiological profile of clonorchis sinensis infection in one community, Guangdong, People's Republic of China," Parasites \& Vectors, vol. 6, 2013.

[6] C. H. Kim, Y. E. Na, N. M. Kim, D. W. Shin, and D. Y. Chang, "Intestinal parasite and clonorchis sinensis infection among the inhabitants in the upper stream of Taechong Dam, Kumgang (River)," Korean Journal of Parasitology, vol. 32, no. 4, pp. 207214, 1994.

[7] T. S. Kim, S. H. Cho, S. Huh et al., "A nationwide survey on the prevalence of intestinal parasitic infections in the Republic of Korea, 2004," Korean Journal of Parasitology, vol. 47, no. 1, pp. 37-47, 2009.

[8] Korea Centers for Disease Control and Prevention and Korea National Institute of Health, "National survey of the prevalence of intestinal parasitic infections in Korea, 2012," The 8th Report, Korea Centers for Disease Control and Prevention and Korea National Institute of Health, Osong, Chungcheongbuk-do, Republic of Korea, 2013.

[9] B. S. Seo, S. H. Lee, S. Y. Cho et al., "An epidemiologic study on clonorchiasis and metagonimiasis in riverside areas in Korea," Korean Journal of Parasitology, vol. 19, no. 2, pp. 137-150, 1981.

[10] S.-H. Cho, K.-Y. Lee, B.-C. Lee et al., "Prevalence of clonorchiasis in southern endemic areas of Korea in 2006," Korean Journal of Parasitology, vol. 46, no. 3, pp. 133-137, 2008.

[11] H.-K. Kim, H.-I. Cheun, B.-S. Cheun et al., "Prevalence of clonorchis sinensis infections along the five major rivers in Republic of Korea, 2007," Osong Public Health and Research Perspectives, vol. 1, no. 1, pp. 43-49, 2010.
[12] H. J. Rim, "Epidemiology and control of clonorchiasis in Korea," Southeast Asian Journal of Tropical Medicine and Public Health, vol. 28, supplement 1, pp. 47-50, 1997.

[13] J. H. Lim, E. Mairiang, and G. H. Ahn, "Biliary parasitic diseases including clonorchiasis, opisthorchiasis and fascioliasis," Abdominal Imaging, vol. 33, no. 2, pp. 157-165, 2008.

[14] H. A. Carpenter, "Bacterial and parasitic cholangitis," Mayo Clinic Proceedings, vol. 73, no. 5, pp. 473-478, 1998.

[15] M. B. Qian, P. Yap, Y. C. Yang et al., "Accuracy of the Kato-Katz method and formalin-ether concentration technique for the diagnosis of clonorchis sinensis, and implication for assessing drug efficacy," Parasites \& Vectors, vol. 6, no. 1, 2013.

[16] H. G. Kim, J. Han, M.-H. Kim et al., "Prevalence of clonorchiasis in patients with gastrointestinal disease: a korean nationwide multicenter survey," World Journal of Gastroenterology, vol. 15, no. 1, pp. 86-94, 2009.

[17] G. S. Lee, I. S. Cho, Y. H. Lee et al., "Epidemiological study of clonorchiasis and metagonimiasis along the Geum-gang (River) in Okcheon-gun (County), Korea," Korean Journal of Parasitology, vol. 40, no. 1, pp. 9-16, 2002.

[18] K. J. June, S. H. Cho, W. J. Lee, C. Kim, and K.-S. Park, "Prevalence and risk factors of clonorchiasis among the populations served by primary healthcare posts along five major rivers in South Korea," Osong Public Health and Research Perspectives, vol. 4, no. 1, pp. 21-26, 2013.

[19] D.-S. Park, S.-J. Na, S. H. Cho, K. J. June, Y.-C. Cho, and Y.H. Lee, "Prevalence and risk factors of Clonorchiasis among residents of riverside areas in Muju-gun, Jeollabuk-do, Korea," Korean Journal of Parasitology, vol. 52, no. 4, pp. 391-398, 2014.

[20] R. Zhang, S. Gao, Y. Geng et al., "Epidemiological study on Clonorchis sinensis infection in Shenzhen area of Zhujiang delta in China," Parasitology Research, vol. 101, no. 1, pp. 179$183,2007$.

[21] W.-C. Liao, H.-P. Wang, H.-M. Chiu, C.-Y. Chang, and J.-T. Lin, "Multiple hepatic nodules: Rare manifestation of clonorchiasis," Journal of Gastroenterology and Hepatology (Australia), vol. 21, no. 9, pp. 1497-1500, 2006. 


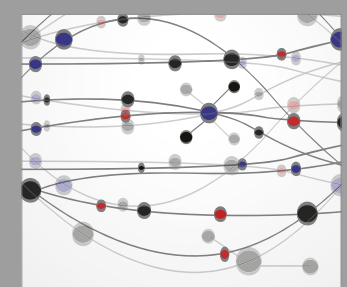

The Scientific World Journal
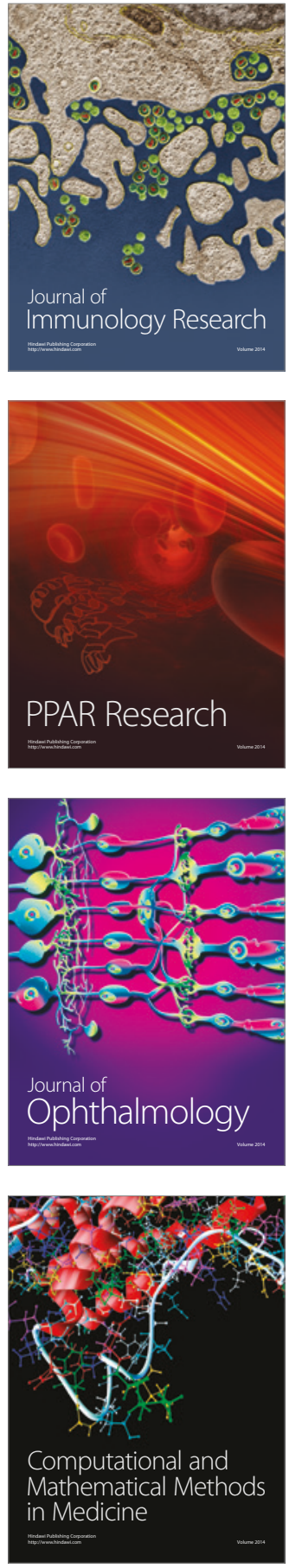

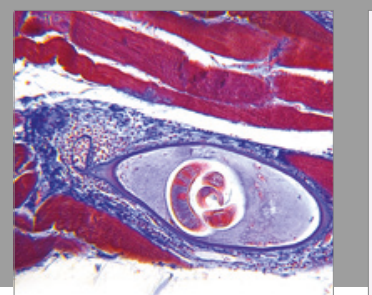

Gastroenterology Research and Practice
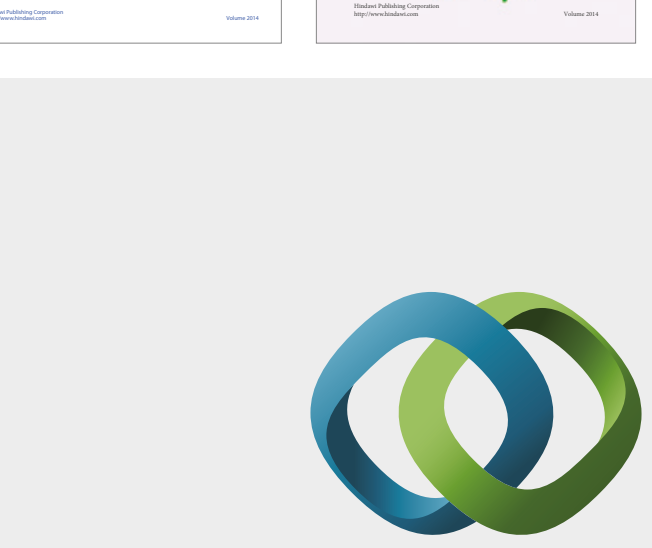

\section{Hindawi}

Submit your manuscripts at

https://www.hindawi.com
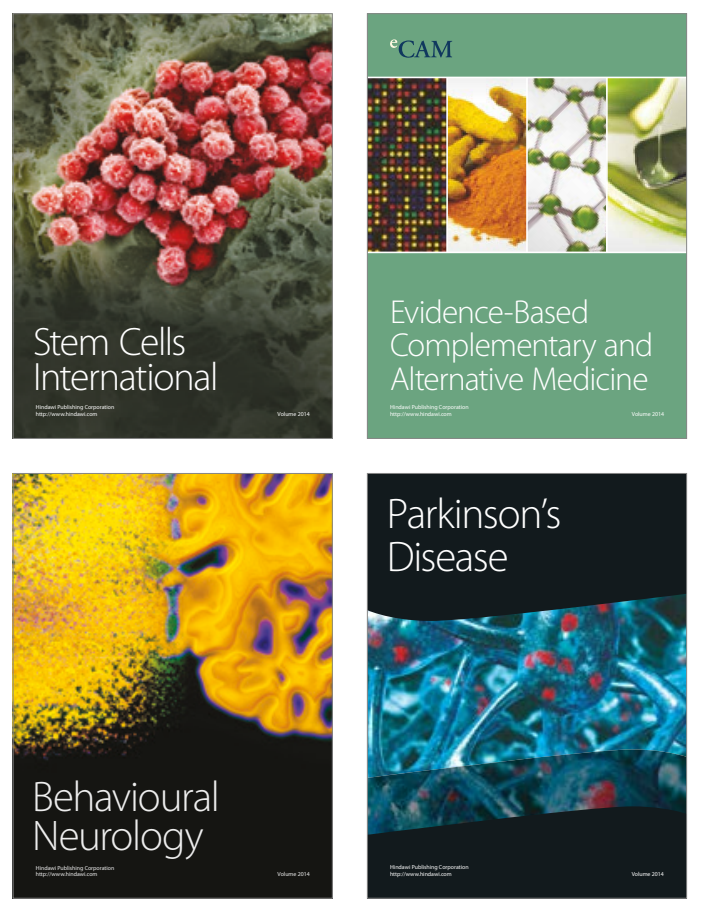
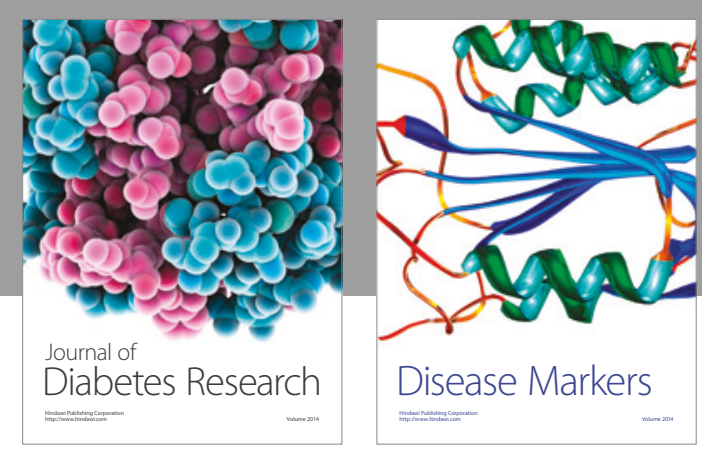

Disease Markers
